\title{
The Economic Burden of Unrecognized Vasodepressor Syncope
}

Hugh CALKInS, M.D., MARK BYRNE, M.D., RAFEL El-ATASSI, M.D., STEVE KALBFLEISCH, M.D., JONATHAN J. LANGBERG, M.D., FRED MORADY, M.D., Ann Arbor, Michigan

BACKGROUND: The objective of this study was to describe the cost of prior diagnostic evaluation in patients referred for evaluation of syncope whose history was typical of vasodepressor syncope.

METHODS AND RESULTS: Thirty consecutive patients who were referred for evaluation of syncope of undetermined origin and whose history was highly suggestive of vasodepressor syncope participated in this study. These 30 patients represented $19 \%$ of 158 patients referred for evaluation of syncope during the period of enrollment. All patients had positive results of an upright-tilt test, confirming the diagnosis of vasodepressor syncope. At the time of evaluation, the type and results of all diagnostic tests that had been performed prior to referral were recorded for each patient. The cost of diagnostic testing was then determined based on the 1991 cost of these tests at the University of Michigan Medical Center.

A mean of $4 \pm 2$ major diagnostic tests were performed before referral to the University of Michigan Medical Center. The mean and median costs of diagnostic testing per patient prior to referral were $\$ 3,763 \pm 3,820$ and $\$ 2,678$ (range: 0 to $\$ 16,606$ ) respectively. Six patients underwent no major diagnostic tests prior to referral and, therefore, the cost of major diagnostic testing was zero in these patients. In the remaining patients, the mean and median costs of diagnostic testing per patient were $\$ 4,704 \pm 3,713$ and $\$ 3,777$ (range: $\$ 1,025$ to $\$ 16,606$ ) respectively.

CONCLUSIONS: The results of this study demonstrate that a diagnosis of vasodepressor syncope can be established clinically in approximately

From the Division of Cardiology, The University of Michigan Medical Center, Ann Arbor, Michigan.

Requests for reprints should be addressed to Hugh Calkins, M.D., Division of Cardiology, The Johns Hopkins Hospital, Carnegie Room 530, 600 North Wolfe Street, Baltimore, Maryland 21287.

Manuscript submitted September 17, 1992, and accepted in revised form February 8, 1993.
$20 \%$ of patients referred to a university hospital for evaluation of syncope of undetermined origin. Failure to recognize the clinical features of vasodepressor syncope in these patients resulted in up to $\$ 16,000$ of unnecessary diagnostic testing. A greater awareness of the clinical features of vasodepressor syncope may, therefore, result in significant economic savings.

V asodepressor syncope is extremely common, accounting for up to $40 \%$ of cases of syncope [1-5]. Typical vasodepressor syncope can be diagnosed based on historical features of the episode [1-5]. We have noted that many patients referred for evaluation of syncope already have undergone extensive diagnostic testing despite a history strongly suggestive of vasodepressor syncope. In these patients, the failure to recognize the clinical features of vasodepressor syncope led to an expensive diagnostic evaluation that unnecessarily increased the cost of medical care.

The purpose of this study was to describe the cost of prior diagnostic evaluation in patients referred for the evaluation of syncope whose history was typical of vasodepressor syncope. Our aim is to highlight the potential savings that would result from a heightened awareness of the clinical features of vasodepressor syncope.

\section{PATIENTS AND METHODS}

Thirty consecutive patients who were referred to the University of Michigan for evaluation of syncope of undetermined origin between September 1989 and July 1991 and whose history was highly suggestive of vasodepressor syncope participated in this study. These 30 patients represented $19 \%$ of 158 patients referred to the University of Michigan for evaluation of syncope during this time period. Each patient underwent an upright-tilt-table test to confirm the diagnosis of vasodepressor syncope [6-12]. At the time of evaluation, the type and results of all diagnostic tests that had been performed prior to referral were recorded for each patient.

There were 16 men and 14 women and their mean age was $39 \pm 16$ years ( \pm standard deviation; range: 


\begin{tabular}{|lcc|}
\hline $\begin{array}{l}\text { TABLE I } \\
\text { Symptoms in 30 Patients With Vasodepressor Syncope }\end{array}$ \\
\hline \multicolumn{1}{|c}{ Symptom } & $\begin{array}{c}\text { No. of } \\
\text { Patients }\end{array}$ & $\begin{array}{c}\text { Prevalence } \\
\text { (\%) }\end{array}$ \\
\hline Identifiable precipitant & 10 & 33 \\
\hline Upright position & 28 & 93 \\
\hline Prodromal symptoms & 30 & 100 \\
Nausea & 19 & 63 \\
Warmth & 21 & 70 \\
Lightheadedness & 21 & 70 \\
Constriction of visual fields & 14 & 47 \\
Epigastric discomfort & 6 & 20 \\
Premonition of syncope & 25 & 83 \\
\hline Pallor & 20 & $100^{\star}$ \\
\hline No injury & 18 & 60 \\
\hline Residual symptoms & 30 & 100 \\
Weakness & 25 & 83 \\
Lightheadedness & 12 & 40 \\
Nausea & 12 & 40 \\
Diaphoresis & 17 & 57 \\
No confusion & 27 & 90 \\
\hline
\end{tabular}

*Pallor was observed in each of the 20 patients who had a witnessed syncopal episode.

17 to 71 years). Twenty-six patients had no history of structural heart disease, 2 patients had mitral valve prolapse, and 2 patients had a history of hypertension. Each patient had experienced one or more episodes of syncope. Twelve patients had greater than 10 syncopal episodes. The mean number of syncopal episodes in the remaining patients was $4 \pm 2$. Four of these patients had experienced only a single episode of syncope.

\section{Clinical Diagnosis of Vasodepressor Syncope}

At the time of evaluation, each patient was interviewed using a standard questionnaire. The questionnaire was designed to determine the likelihood of vasodepressor syncope by reviewing the past medical history and the historical details of the syncopal episodes. The patients were asked a series of specific questions to identify precipitating factors, position and activity prior to syncope, color and appearance during syncope, resultant injury, and the type and duration of prodromal and residual symptoms. In addition, patients were asked to give a detailed account of their two most recent episodes of syncope. Based on their response to this questionnaire, each patient in this study was clinically diagnosed as having vasodepressor syncope. Symptoms that were believed to be suggestive of vasodepressor syncope included: (1) the presence of an obvious precipitant such as pain, fear, emotional stress, alcohol, or a large meal; (2) syncope occurring only in the standing or seated position; (3) the presence of typical prodromal symptoms including nausea, warmth, lightheadedness, weakness, diaphoresis, constriction of the visual fields, epigastric discomfort, and/or a sensation of an impending faint for at least 5 seconds prior to syncope; (4) the observation by a bystander of pallor during syncope; (5) the absence of significant injury; (6) the presence of typical residual symptoms including weakness, lightheadedness, nausea, and fatigue lasting minutes to hours; and (7) the absence of confusion in the recovery period [1-5,13-16]. Patients were diagnosed as having vasodepressor syncope if they had a normal electrocardiogram, no significant history of cardiovascular disease, and the presence of signs or symptoms from five or more categories. The frequency with which each of these symptoms was reported by the patients is shown in Table $I$.

\section{Upright-Tilt Testing}

Upright-tilt testing was performed in the fasting state. The patients were connected to a standard cardiac monitor and an automatic sphygmomanometer, and were placed on a motorized table with a foot board for weight bearing. Passive upright tilt to $70^{\circ}$ was performed in the drug-free state for 15 minutes. If the patients' symptoms were not reproduced, upright tilt was repeated for 15 minutes during an infusion of $4 \mu \mathrm{g} / \mathrm{min}$ of isoproterenol. The patients' blood pressure was recorded in the supine position, 1 minute following tilt, and thereafter at 5minute intervals. If symptoms developed, blood pressure was recorded at 1-minute intervals. The patients' electrogram was continuously monitored during the procedure. A rhythm strip was recorded at 5-minute intervals, then continuously with the development of symptoms. A positive response to upright tilt was defined as the development of presyncope or syncope associated with hypotension and an absolute or relative bradycardia. A relative bradycardia was defined as greater than a 5 beat per minute decrease in heart rate from the peak achieved heart rate. The test was terminated if the patient developed chest pain or severe discomfort. There were no complications during or following upright-tilt testing.

Each patient had a vasodepressor response to upright-tilt testing that reproduced their clinical symptoms. Five patients developed a vasodepressor response to upright tilt alone and 25 patients developed a vasodepressor response to upright tilt in conjunction with an isoproterenol infusion.

\section{Clinical Follow-Up}

If the frequency and severity of symptoms were believed to mandate therapy, patients were treated 


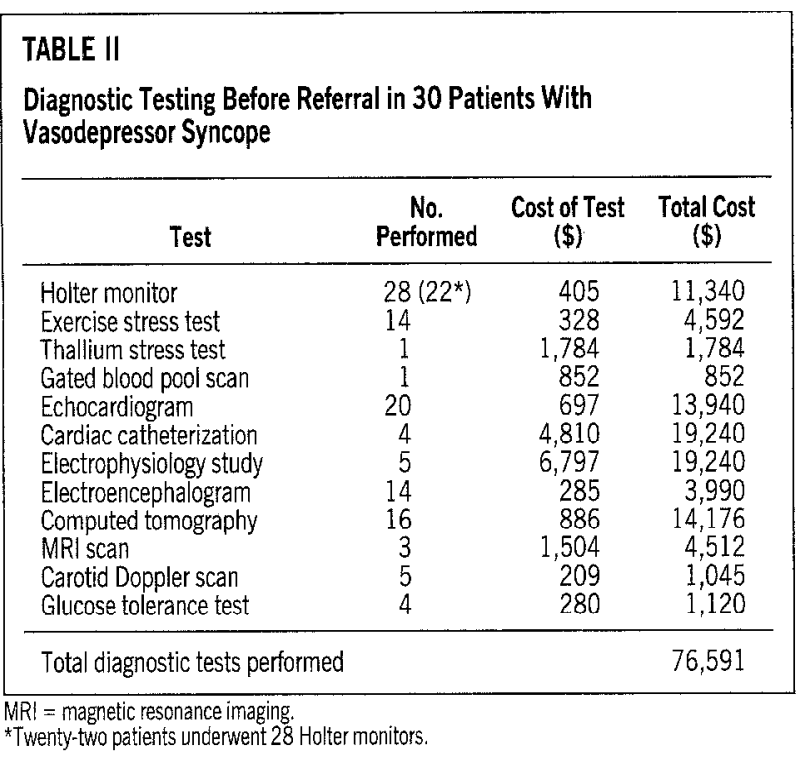

with pharmacologic or pacemaker therapy. The type of therapy was individualized and the efficacy of the therapy was guided by follow-up upright-tilt tests. Each patient was followed for a minimum of 6 months to determine the frequency and type of recurrent symptoms.

Twenty-eight patients were treated with pharmacologic $(n=26)$, pacemaker $(n=1)$, or combined pharmacologic and pacemaker therapy $(n=1)$ for prevention of recurrent syncope. Long-term therapy was not believed to be necessary in two patients who had sustained only a single episode of syncope that was not associated with significant injury. Seventeen patients were treated long term with $\beta$ blockers, 7 with disopyramide, 1 with aminophylline, and 2 with combination therapy. Twenty-nine of the 30 patients were contacted at a mean of $17 \pm 7$ months following their initial evaluation. Six patients have experienced presyncope but no patient has had recurrent syncope during follow-up.

\section{Cost of Prior Syncope Evaluation}

The cost of major diagnostic tests performed as part of the syncope evaluation was estimated by determining the number and type of major diagnostic tests that had been obtained prior to referral. These data were obtained by interviewing the patients at the time of their evaluation and also by contacting their referring physicians. Major diagnostic tests included cardiac catheterization, electrophysiology tests, echocardiography, Holter monitoring, computerized tomographic (CT) scans of the head, electroencephalograms (EEGs), magnetic

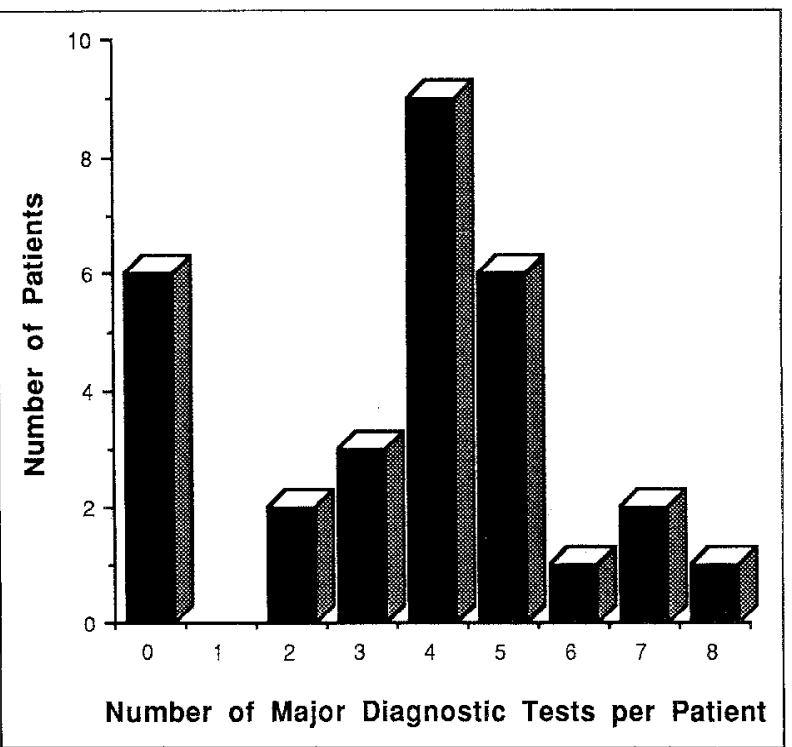

Figure 1. The number of major diagnostic tests performed per patient.

resonance imaging (MRI), glucose tolerance tests, and carotid Doppler studies. The cost of diagnostic testing was then determined based on the 1991 cost of these tests at the University of Michigan Medical Center. The cost of these tests included both the hospital charges and the physician's interpretation fee. In this analysis, we did not include the cost of office visits, consultations, emergency room visits, and hospitalizations.

\section{RESULTS}

\section{Previous Evaluation}

A mean of $4 \pm 2$ major diagnostic tests were performed before referral to the University of Michigan Medical Center. The number and type of diagnostic tests that were performed are shown in Table II. Holter monitoring was the most frequently obtained diagnostic test, followed by echocardiograms, CT scans of the head, and exercise tolerance tests. More than half of the patients underwent each of these tests. The distribution of the number of major diagnostic tests that were performed in each patient is shown in Figure 1. Six patients were directly referred for evaluation of suspected vasodepressor syncope and underwent no major diagnostic tests. The remaining 24 patients underwent a mean of $5 \pm 2$ major diagnostic tests prior to referral. Abnormalities detected using these tests included mitral valve prolapse in two patients, mild mitral regurgitation in one patient, left ventricular hypertrophy in one patient, lacunar cerebral infarcts in one patient, paroxysmal atrial fibrillation in one patient, wandering atrial pacemaker in one 


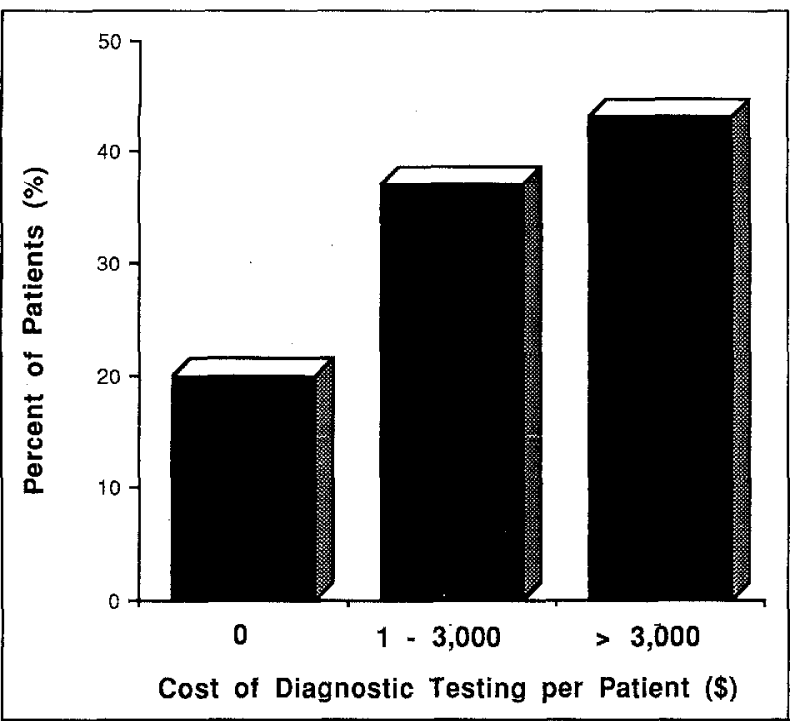

Figure 2. The cost of major diagnostic testing per patient. Shown on the horizontal axis is the cost of major diagnostic testing. Shown on the vertical axis are the percentage of patients in each cost group.

patient, sinus bradycardia in one patient, and nonspecific EEG abnormalities in three patients. In no patient were these abnormalities thought to be diagnostic of the cause of syncope [2].

\section{Cost of Diagnostic Testing}

The cost of each diagnostic test, the total number performed, and the associated total expenditure for each type of test are shown in Table II. Electrophysiology tests were the most expensive diagnostic tests followed by cardiac catheterizations, thallium stress tests, and MRI scans.

The mean and median costs of diagnostic testing per patient prior to referral were $\$ 3,763 \pm 3,820$ and $\$ 2,678$ (range: 0 to $\$ 16,606$ ) respectively. The distribution of the cost for diagnostic testing is shown in Figure 2. The cost of prior evaluation exceeded $\$ 3,000$ in more than one third of the patients. Six patients underwent no major diagnostic tests prior to referral and, therefore, the cost was zero in these patients. In the remaining patients, the mean and median costs of diagnostic testing per patient were $\$ 4,704 \pm 3,713$ and $\$ 3,777$ (range: $\$ 0,025$ to $\$ 16,606$ ) respectively.

\section{COMMENTS}

\section{Main Findings}

An alarming rise in the cost of medical care in the United States has emphasized the importance of cost containment in the health care industry [17]. One method by which cost containment can be achieved is by the elimination of unnecessary diagnostic tests [18]. The results of this study demonstrate that a significant proportion of patients who have vasodepressor syncope may be undergoing thousands of dollars' worth of unnecessary diagnostic testing. Approximately $20 \%$ of patients referred to our institution for evaluation of syncope had a clinical history typical of vasodepressor syncope, with this diagnosis being confirmed by upright-tilt testing. Despite having a condition that was diagnosable on a clinical basis, the majority of these patients underwent extensive neurologic and/or cardiovascular evaluation prior to referral. Given the fact that syncope is a common problem, accounting for $3 \%$ of emergency room visits and up to $6 \%$ of hospital admissions $[1,19]$, the results of this study suggest that an increased awareness of the typical clinical features of vasodepressor syncope could eliminate unnecessary and expensive diagnostic testing in many patients, resulting in substantial cost savings.

\section{Clinical Diagnosis of Vasodepressor Syncope}

The diagnosis of vasodepressor syncope depends on the recognition of historical features of the syncopal episode [1-5,13,14]. Vasodepressor syncope typically occurs in the standing and/or seated position and is triggered by pain, fear, emotional stress, alcohol, and/or a large meal. Prior to syncope, the patient experiences prodromal symptoms such as nausea, warmth, lightheadedness, weakness, diaphoresis, constriction of visual fields, epigastric discomfort, and/or a sensation of an impending faint. These symptoms usually persist for at least several seconds prior to syncope. While unconscious, the patient appears pallid. After regaining consciousness, the patient typically is not confused but experiences residual symptoms such as weakness, lightheadedness, nausea, and/or fatigue, which may last for minutes to hours.

The relative importance of each of these symptoms in establishing the diagnosis of vasodepressor syncope is uncertain. Some studies have required an identifiable precipitating factor to establish a diagnosis of vasodepressor syncope [2], whereas others have based the diagnosis on a general clinical impression of the patient's history $[1,3,4]$. The studies that have required identification of a precipitant report a lower incidence of vasodepressor syncope and a greater incidence of syncope of undetermined origin as compared to the studies in which the diagnosis was based on the overall clinical impression. In this study, the diagnosis of vasodepressor syncope did not require identification of a precipitant, but was based on the presence of a constellation of signs and symptoms suggestive of vasodepressor syncope. In fact, a precipitant was identified in only one third of patients in this study. Yet, the response 
of the patients to upright-tilt testing and their response to therapy support the diagnosis of vasodepressor syncope. These findings suggest that criteria for vasodepressor syncope that require identification of a precipitating factor may be too rigid.

The precise sensitivity and specificity of each of the individual symptoms that commonly occur during vasodepressor syncope have not been determined. Although prodromal symptoms, residual symptoms, and pallor were uniformly present in the patients in this study ( $100 \%$ sensitivity), the specificity of these clinical features in distinguishing vasodepressor syncope from other causes of syncope is uncertain. Nevertheless, given the low cost of upright-tilt testing and the prevalence of vasodepressor syncope, if these symptoms are present, it may be appropriate to perform an upright-tilt test before embarking on an extensive neurologic and/or cardiac evaluation.

\section{Sensitivity, Specificity, and Reproducibility of Up- right-Tilt Testing}

No prior study has evaluated the sensitivity of upright-tilt testing in patients with vasodepressor syncope, the diagnosis of which was based on clinical symptoms. However, previous studies have evaluated the sensitivity and specificity of upright-tilt testing in patients with syncope of unknown origin. In the absence of isoproterenol, upright-tilt testing results in a positive (vasodepressor) response in $8 \%$ to $66 \%$ of patients with syncope and less than $10 \%$ of controls [6-12,20-22]. The use of isoproterenol in conjunction with upright-tilt testing results in a positive response in $78 \%$ to $82 \%$ of patients with syncope $[6,7,11,12,21,22]$. The precise specificity of upright-tilt testing in conjunction with an isoproterenol infusion remains uncertain. Kapoor and Brant [21] recently reported a positive response to upright-tilt in conjunction with an isoproterenol infusion in 21 of 40 control patients. In contrast, studies by Sheldon and Killam [20], Almquist et al [6], and Grubb et al [12] reported positive responses to upright tilt in conjunction with an isoproterenol infusion in 0 of 15,2 of 18 , and 0 of 6 control patients.

The uncertain specificity of upright-tilt testing in conjunction with an isoproterenol infusion has little impact on the findings of this study because the diagnosis of vasodepressor syncope in this study was established on a clinical basis. The accuracy of this clinical diagnosis of vasodepressor syncope is supported, but not proved, by both the presence of a positive response to upright-tilt testing (with reproduction of clinical symptoms in all patients) as well as their response to therapy.

\section{Economic Benefit of a Directed Approach to Vaso- depressor Syncope}

The majority of patients in this study underwent extensive diagnostic testing prior to referral. This suggests either an underappreciation by referring physicians of the clinical features of vasodepressor syncope or a reliance on excessively rigid clinical criteria for establishment of a diagnosis of vasodepressor syncope. The extensive testing performed in these patients also suggests that physicians may be uncomfortable diagnosing "syncope of unknown origin" without performing several screening tests. The findings of previous studies that have demonstrated the low diagnostic yield and cost ineffectiveness of these diagnostic tests appear to have had little impact $[2,5,19,22-26]$. This most likely reflects both the perceived need by physicians and patients to establish a diagnosis and the widespread availability of diagnostic testing.

Within the entire group of patients in this study, two subgroups emerged. The first, and larger group, underwent extensive evaluation prior to referral. In these patients, an average of five major diagnostic tests were performed at a cost of nearly $\$ 5,000$ per patient, with the cost in individual patients being as high as $\$ 16,000$. The second group of patients were referred directly for evaluation and demonstrate the potential economic savings of a more directed approach to vasodepressor syncope. In these patients, the diagnosis of vasodepressor syncope was suspected on a clinical basis and was confirmed with upright-tilt testing. The cost of diagnostic testing in these patients was limited to the cost of an upright-tilt test ( $\$ 453)$, thereby saving the cost of additional diagnostic studies.

\section{Limitations}

There are two limitations to this study. First, the type of patients referred to a university medical center depends on local referral patterns and the sophistication of referring physicians. Although approximately $20 \%$ of the patients referred to our hospital had a history typical of vasodepressor syncope, this percentage may be higher or lower at other medical centers. However, the University of Michigan is a typical referral center, with patients being referred by a wide range of physicians, including general practitioners, internists, and cardiologists, and there is no reason to believe that our population of patients differs significantly from that at other large medical centers.

A second limitation of this study is that only the 
costs of major diagnostic tests were included for analysis. Minor diagnostic tests, the cost of consultations, and hospital charges were not included, in part because of the difficulty in accurately determining these costs. Therefore, this study underestimates the potential cost savings of a heightened awareness of the clinical features of vasodepressor syncope.

A third limitation of this study is that no gold standard exists for establishing a diagnosis of vasodepressor syncope. Although the criteria used to establish a diagnosis of vasodepressor syncope in this study are conservative, these criteria have not been independently validated.

\section{Clinical Implications}

Based on the results of this study, the following approach to patients with syncope can be recommended. Patients with a history typical of vasodepressor syncope should have the diagnosis established on a clinical basis at the time of their initial evaluation. If the patient has a normal physical examination result, a normal electrocardiogram, and no history of cardiac disease, and the syncopal episode was associated with a precipitant, a prodromal period, and no significant injury, no further evaluation or therapy may be necessary. If prophylactic pharmacologic therapy is appropriate to prevent recurrences of syncope, upright-tilt testing may be useful in guiding further therapy [12]. Several recent studies have demonstrated that pharmacologic agents such as $\beta$ blockers and disopyramide may be helpful in preventing recurrent vasodepressor syncope $[12,27]$.

Upright-tilt testing is appropriate as the initial diagnostic test in patients without clinical evidence of heart disease whose symptoms are consistent with, but not entirely typical of, vasodepressor syncope. For example, a patient may have had typical residual symptoms but no prodromal symptoms at the time of syncope. If the patient's clinical symptoms are reproduced, further neurologic and/or cardiovascular evaluation can be avoided.

Patients whose history is not suggestive of vasodepressor syncope should be evaluated in a standard fashion, with the type and extent of diagnostic testing guided by the history, physical examination, and electrocardiogram [19]. For instance, the patient with a prior myocardial infarction and impaired ventricular function who experiences syncope without prodromal or residual symptoms and whose electrocardiogram demonstrates a bundlebranch block should be considered for electrophysiologic testing because the likelihood of detecting a potentially life-threatening arrhythmia such as ventricular tachycardia in this type of patient is high [28].

\section{ACKNOWLEDGMENT}

We thank Vanessa Sims and Elaine Lowery for their secretarial help in the preparation of this manuscript.

\section{REFERENCES}

1. Day SC, Cook EF, Funkenstein H, Goldman L. Evaluation and outcome of emergency room patients with transient loss of consciousness. Am J Med 1982; 73: 15-23.

2. Kapoor WN. Evaluation and outcome of patients with syncope. Medicine (Baltimore) 1990; 69: 160-75.

3. Wayne HH. Syncope. Physiological considerations and an analysis of the clinical characteristics in 510 patients. Am J Med 1961; 30: 418-38.

4. Martin GJ, Adams SL, Martin HG, Matthews J, Zull D, Scanion PJ. Prospective evaluation of syncope. Ann Emerg Med 1984; 13: 499-504.

5. Kapoor WN, Karpf M, Wieand S, Peterson JR, Levey GS. A prospective evaluation and follow-up of patients with syncope. N Engl J Med 1983; 309: 197-204. 6. Almquist A, Goldenberg IF, Milstein S, et al. Provocation of bradycardia and hypotension by isoproterenol and upright posture in patients with unexplained syncope. N Engi J Med 1989; 320: 346-51.

7. Milstein S, Reyes W, Benditt DG. Upright body tilt for evaluation of patients with recurrent, unexplained syncope. PACE Pacing Clin Electrophysiol 1989; 12 : 117-24.

8. Kenny RA. Bayliss J, Ingram A, Sutton R. Head-up tilt: a useful test for investigating unexplained syncope. Lancet 1986; 1: 1352-5.

9. Benditt DG, Remole S, Bailin S, Dunnigan A, Asso A, Milstein S. Tilt table testing for evaluation of neurally-mediated (cardioneurogenic) syncope: rationale and proposed protocols. PACE Pacing Clin Electrophysiol 1991; 14: 1528-37.

10. Waxman MB, Yao L, Cameron DA, Waid RW, Roseman J. Isoproterenol induction of vasodepressor-type reaction in vasodepressor-prone persons. Am J Cardiol 1989; 63: 58-65.

11. Pongiglione G, Fish FA, Strasburger JF, Benson W. Heart rate and blood pressure response to upright tilt in young patients with unexplained syncope. $J$ Am Coll Cardiol 1990; 16: 165-70.

12. Grubb BP, Temesy-Armos P, Hahn H, Elliott L. Utility of upright tilt-table testing in the evaluation and management of syncope of unknown origin. Am J Med 1991; 90: 6-10.

13. Weissler AM, Warren JV, Vasodepressor syncope. Am Heart J 1959; 57: 786-94.

14. Engel GL. Fall in arterial blood pressure. In: Aring CD, editor. Fainting: physiological and psychological considerations. 1st ed. Springfield, IL: Charles C Thomas, 1950: 6-35.

15. Engel GL. Psychologic stress, vasodepressor (vasovagal) syncope, and sudden death. Ann Intern Med 1978; 89: 403-12.

16. Ruetz PP, Johnson SA, Callahan $R$, Meade RC, Smith JJ. Fainting: a review of its mechanisms and a study of blood donors. Medicine (Baltimore) 1967; 46: 363-84.

17. McNerney WJ. Control of health-care costs in the 1980's. N Engl J Med 1980; 303: 1088-95.

18. Eisenberg JM, Rosoff AJ. Physician responsibility for the cost of unnecessary medical services. N Engl J Med 1978; 299: 76-80.

19. Gendelman HE, Linzer M, Gabelman M, Smoller S, Scheuer J. Syncope in a general hospital patient population. NY State J Med 1983; 83: 1161-5.

20. Sheldon $R$, Killam S. Methodology of isoproterenol-tilt table testing in patients. J Arn Coll Cardiol 1992; 19: 773-9.

21. Kapoor WN, Brant N. Evaluation of syncope by upright tilt testing with isoproterenol. Ann Intern Med 1992; 116: 358-63.

22. Davis TL, Freemon FR. Electroencephalography should not be routine in the evaluation of syncope in adults. Arch Intern Med 1990; 150: 2027-9.

23. Sheldon R, Splawinski J, Killam S. Reproducibility of isoproterenol tilt-table tests in patients with syncope. Am J Cardiol 1992; 69: 1300-5.

24. Kapoor W. Karpf M, Levey GS. Issues in evaluating patients with syncope. 
Ann Intern Med 1984; 100: 755-9.

25. Eagle KA, Black HR. The impact of diagnostic tests in evaluating patients with syncope. Yale J Biol Med 1983; 56: 1-8.

26. Kapoor WN, Karpf M, Maher Y, Miller RA, Levey GS. Syncope of unknown origin. The need for a more cost-effective approach to its diagnostic evaluation. JAMA 1982; 247: 2687-91.
27. Milstein S, Buetikofer J, Dunnigan A, Benditt DG, Gornick C, Reyes WJ. Usefuiness of disopyramide for prevention of upright tilt-induced hypotensionbradycardia. Am J Cardiol 1990; 65: 1339-44.

28. Krol RB, Morady F, Flaker GC, et al. Electrophysiologic testing in patients with unexplained syncope: clinical and noninvasive predictors of outcome. J Am Coll Cardiol 1987; 10: 358-63. 